\title{
Diversidad faunística y potenciales agentes tafonómicos en el Chaco árido (Córdoba, Argentina): una aproximación preliminar con cámaras trampa
}

\author{
M. Paula Weihmüller, Thiago Costa, Lucila B. Castro y Fernando R. Barri \\ Recibido 14 de noviembre 2020. Aceptado 25 de marzo 2021
}

\begin{abstract}
RESUMEN
El objetivo de este trabajo consistió en identificar las principales especies animales que actúan sobre carcasas de ungulados de gran porte en un área cercana al extremo austral de las Salinas Grandes de Córdoba y su potencial como agentes tafonómicos. Con este fin, se realizaron tres monitoreos con cámaras trampa sobre carcasas de Bos taurus y Lama guanicoe y se completaron 194 días-trampa entre noviembre de 2017 y mayo de 2019. Además, se efectuaron observaciones preliminares sobre desarticulación, dispersión y trazas generadas por distintos agentes tafonómicos sobre las carcasas, a fin de contribuir a nuestro entendimiento sobre los procesos naturales de formación de conjuntos zooarqueológicos en la región. Los monitoreos permitieron registrar 5869 animales e identificar 30 especies. Nuestro registro sugiere que las modificaciones observadas en las carcasas de la región pueden ser producidas por una variedad de animales que excede a los habitualmente considerados en estudios zooarqueológicos. Las especies con mayor potencial de generar modificaciones tafonómicas fueron un mamífero autóctono, Lycalopex griseus, y dos introducidos, Sus scrofa y Canis lupus familiaris. La actividad de L. griseus fue registrada tanto en las etapas iniciales de descomposición como en una carcasa esqueletizada. S. scrofa y C. lupus familiaris se presentaron en las etapas inmediatamente post mortem y fueron los principales responsables de la dispersión y destrucción ósea. Entre las aves, se destaca la presencia de accipitriformes y falconiformes consumiendo las carcasas, pero su potencial para generar trazas detectables en el registro arqueológico es incierto. Por último, se detectó pisoteo por parte de grandes ungulados.
\end{abstract}

Palabras clave: Tafonomía actualista; Córdoba; Agentes tafonómicos; Carroñeros.

\section{Faunal diversity and potential taphonomic agents in the arid Chaco, Córdoba, Argentina: a preliminary approach with camera traps}

\begin{abstract}
The aim of this study was to identify the main animal species that act on carcasses of large ungulates and their potential as taphonomic agents in an area near the southern end of the Salinas Grandes de Córdoba. To this end, three camera-trap surveys were conducted on Bos taurus and Lama guanicoe carcasses, covering 194 trap days between November 2017 and May 2019. In addition, preliminary observations were made of the disarticulation, dispersal, and traces generated by different taphonomic agents acting on the carcasses in order to contribute to the understanding of the natural formation processes of zooarchaeological

María Paula Weihmüller. Instituto de Antropología de Córdoba (IDACOR), Consejo Nacional de Investigaciones Científicas y Técnicas (CONICET), Museo de Antropología, Facultad de Filosofía y Humanidades, Universidad Nacional de Córdoba. Av. Hipólito Yrigoyen 174 (5000), Córdoba, Argentina. E-mail: mpweihmuller@gmail.com

Thiago Costa. IDACOR, CONICET, Museo de Antropología, Facultad de Filosofía y Humanidades, Universidad Nacional de Córdoba. Av. Hipólito Yrigoyen 174 (5000), Córdoba, Argentina. E-mail: thfcosta@gmail.com

Lucila Belén Castro. Instituto de Diversidad y Ecología Animal (IDEA), CONICET, Facultad de Ciencias Exactas, Físicas y Naturales, Universidad Nacional de Córdoba. Av. Vélez Sarsfield 299 (5000), Córdoba, Argentina. E-mail: lucilacastro72@ gmail.com

Fernando Rafael Barri. IDEA, CONICET, Facultad de Ciencias Exactas, Físicas y Naturales, Universidad Nacional de Córdoba. Av. Vélez Sarsfield 299 (5000), Córdoba, Argentina. E-mail: fernando.barri@unc.edu.ar
\end{abstract}

Intersecciones en Antropología 22(1), enero-junio: 125-138. 2021. ISSN-e 1850-373X

https://doi.org/10.37176/iea.22.1.2021.599

Facultad de Ciencias Sociales - UNICEN - Argentina 
assemblages in the region. The monitoring recorded 5869 animals and identified 30 species. The data suggested that modifications observed on carcasses in the region may have been produced by a variety of animals that exceeds those usually considered in zooarchaeological studies. The species with the greatest potential to generate taphonomic modifications were the native mammal, Lycalopex griseus, and two introduced mammals, Sus scrofa and Canis lupus familiaris. The activity of L. griseus is notable for having been recorded both in the early stages of decomposition and on a skeletonized carcass. S. scrofa and C. lupus familiaris activity occurred in the immediate post-mortem stages and was mainly responsible for bone dispersal and destruction. Among birds, accipitriformes and falconiformes were recorded consuming the carcasses, but their potential to generate detectable traces in the archaeological record is uncertain. Finally, trampling by large ungulates was recorded.

Keywords: Actualistic taphonomy; Córdoba; Taphonomic agents; Scavengers.

\section{INTRODUCCIÓN}

Los restos óseos faunísticos retienen información sobre las especies que habitan un ecosistema, la cadena trófica y el reciclaje de nutrientes, entre otros (Behrensmeyer y Miller, 2012). En tanto, el interés por los agentes tafonómicos que intervienen tras la muerte de un animal no es exclusivo de la zooarqueología o la paleontología. De hecho, los estudios tafonómicos actualistas tienen un enorme potencial informativo para la ecología y la conservación, dado que permiten documentar cambios y continuidades en el ecosistema, y así ampliar la profundidad temporal de las investigaciones (Behrensmeyer, Kidwell y Gastaldo, 2000).

En la provincia de Córdoba, las investigaciones zooarqueológicas no se han caracterizado por un componente tafonómico actualista muy fuerte, a diferencia de otras regiones del país, como Pampa (e.g., Gutiérrez et al., 2018) y Patagonia (e.g., Borrero, 1990, 2007). Si bien la mayoría de los trabajos sobre la arqueofauna regional han considerado la acción de agentes transformadores del registro (e.g., Costa 2016), el escaso respaldo con investigaciones actualistas -local o regionalmente situadas- ha obstaculizado la profundización del conocimiento sobre la formación de los conjuntos y sobre las condiciones paleoecológicas subyacentes.

Para comenzar a llenar ese vacío de información nos proponemos realizar un primer acercamiento a los agentes tafonómicos (Erlandson y Moss, 2001) que actúan sobre carcasas de grandes ungulados desde una perspectiva interdisciplinaria, en la que confluyen la tafonomía actualista y la ecología (Behrensmeyer y Miller, 2012), en un área cercana a las Salinas Grandes de Córdoba. El interés por esta región en particular reside en su enorme riqueza en términos de biodiversidad (Costa y Barri, 2018), ya que aloja a gran parte de la fauna autóctona que ha sido identificada en el registro arqueológico de dicha provincia, y esto le otorga potencial como fuente de analogías para interpretar las modificaciones registradas en conjuntos zooarqueológicos.

En concreto, nuestro objetivo consistió en identificar, mediante el uso de cámaras trampa, las principales especies que carroñan carcasas de ungulados de gran porte en la región de estudio y su potencial como agentes tafonómicos, y luego discutir algunas de sus implicancias para los estudios zooarqueológicos regionales (Erlandson y Moss, 2001). El uso de estos dispositivos permite un registro detallado de los agentes involucrados en el carroñeo de las carcasas, algo que algunas décadas atrás solo podía estimarse a partir de las trazas en los huesos (Blumenschine, 1989). Si bien su uso no es completamente ajeno a la arqueología (Forsyth et al., 2014; Cusimano, 2015; Arilla, Rosell y Blasco, 2020), consideramos que, a nivel local, su enorme potencial como herramienta para estudios tafonómicos aún no ha sido explotado. Por último, se presentan observaciones preliminares sobre las modificaciones generadas por los distintos agentes tafonómicos identificados, a fin de contribuir a nuestro entendimiento sobre los procesos de formación de conjuntos zooarqueológicos en la región.

\section{ÁREA DE ESTUDIO}

Este trabajo se desarrolló en un campo privado emplazado en las cercanías de las localidades de Piedrita Blanca y El Chacho (Figura 1A). El campo -de 20.000 ha- colinda al este con la Reserva Arqueológica Provincial de Guasapampa y al sur con el Parque Nacional Traslasierra, dos grandes áreas protegidas por la legislación nacional. El relieve del área es Ilano, con una altura que varía entre 


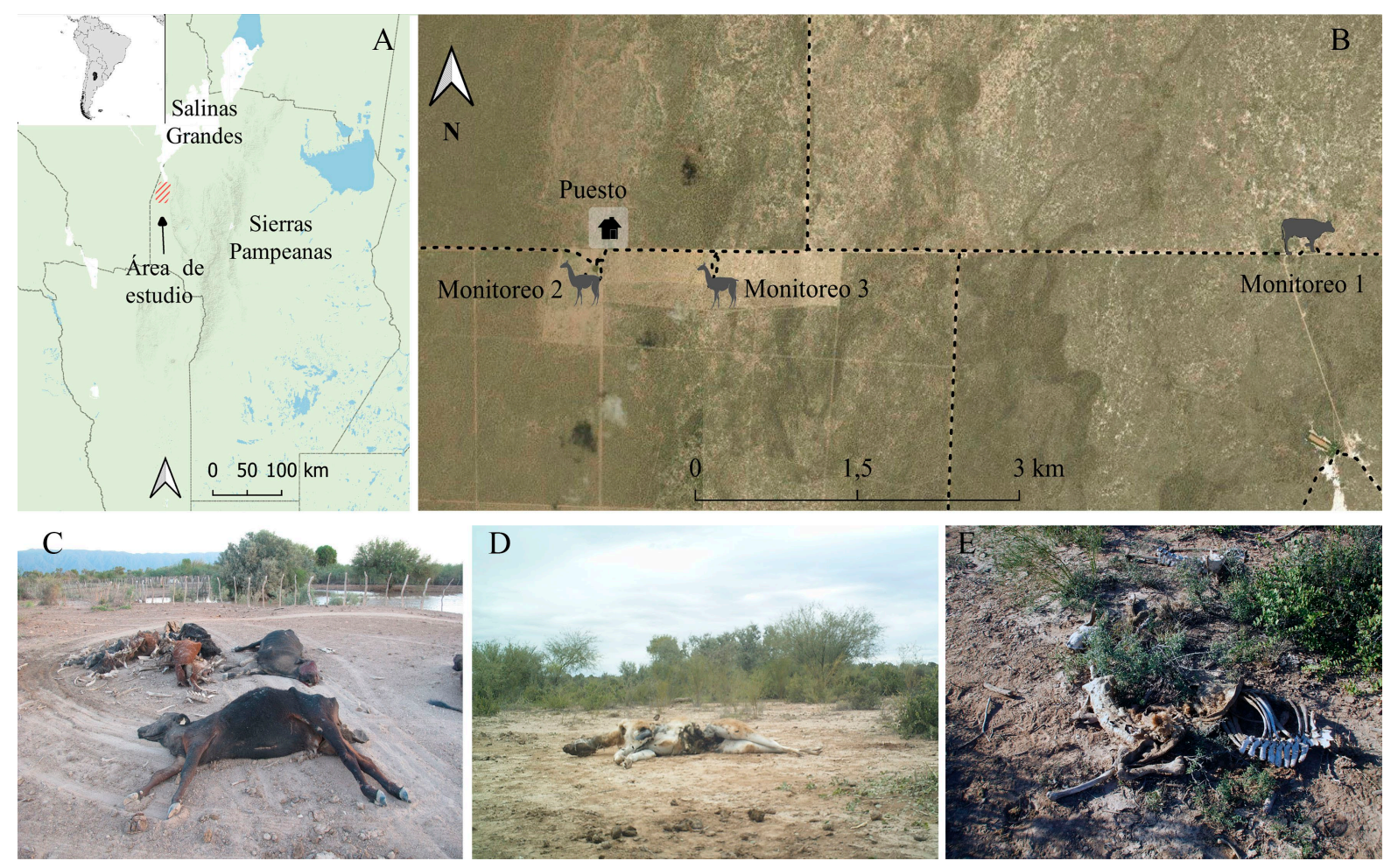

Figura 1. A) Ubicación geográfica del área de estudio. B) Localización de los monitoreos al interior del campo. Estado inicial de las carcasas: C) monitoreo 1; D) monitoreo 2; E) monitoreo 3.

los 200 y 300 msnm, en aumento hacia el este, donde se erigen las sierras de Pocho y Guasapampa (Carignano et al., 2014). Fitogeográficamente, pertenece al Chaco árido, a un bosque de Aspidosperma quebracho-blanco y Prosopis nigra que está siendo desplazado por matorrales y paisajes antropizados (Zak et al., 2008). El clima se caracteriza por grandes amplitudes térmicas diarias y estacionales, con una media de $26{ }^{\circ} \mathrm{C}$ en verano y $16{ }^{\circ} \mathrm{C}$ en invierno. La distribución anual de Iluvias define una época seca (abril-septiembre) y otra húmeda (octubre-marzo), con una media anual de 202,89 mm entre 2012-2020. En cuanto a la fauna, el área se encuentra dentro del dominio central definido por Ringuelet (1961).

\section{METODOLOGÍA}

La recopilación de datos para este trabajo implicó, en primera instancia, la instalación de tres cámaras trampa (Bushnell Trophy Aggressor Camo) en un campo privado en el departamento Minas, Córdoba (Figura 1A), para monitorear una concentración de cinco carcasas de Bos taurus y dos carcasas aisladas de Lama guanicoe (Figura 1C-E). El uso de cámaras trampa para estudios de la vida silvestre se ha incrementado notablemente en los últimos años, ya que es una metodología no invasiva y con alto grado de precisión, que permite determinar las especies presentes en un sitio dado (O'Connell, Nichols y Karanth, 2011). Estos dispositivos detectan movimiento en su área de enfoque mediante un sensor infrarrojo; es decir, cuando la cámara detecta movimiento, captura una fotografía (Muñoz, Beltrami y Osorio, 2017). Esta metodología es útil para monitorear animales difíciles de observar en terreno, e incluirla dentro de un programa tafonómico permite establecer con certeza los agentes involucrados en el carroñeo de un animal (Forsyth et al., 2014; Arilla et al., 2020). Las cámaras fueron programadas para estar activas las 24 horas y capturar una fotografía al activarse, con un retraso de 8-10 minutos entre capturas. El tiempo entre la configuración de la cámara y el último registro fotográfico es considerado como el período efectivo de muestreo del sitio, expresado en días-trampa (Chen et al., 2009).

En la Tabla 1 se resumen las características de los tres monitoreos realizados. El primero corresponde al periodo inmediato post mortem de cinco vacas adultas muertas por el síndrome de vaca caída, a la vera de una represa ubicada a $6 \mathrm{~km}$ del puesto. ${ }^{1}$

La cámara fue instalada el 28 de noviembre de 2017 y retirada 16 días más tarde. Este monitoreo 
fue el más alejado del puesto (Figura 1B) y, en consecuencia, con menor presencia humana. Además, es el monitoreo más cercano al cordón serrano de Guasapampa, sitio con presencia de dormideros de cóndores y otras aves carroñeras. El segundo monitoreo comenzó 15 días post mortem de un individuo de Lama guanicoe hembra de entre uno y dos años, muerto por perros domésticos el 20 de mayo de 2018 en un claro aledaño al puesto y, en tanto, próximo a presencia humana. Este se realizó durante dos períodos consecutivos: el primero comenzó el 31 de mayo de 2018 y se extendió por 24 días, mientras que el segundo comenzó el 25 de junio de 2018 y duró 53 días. El tercer monitoreo se efectuó sobre una carcasa parcialmente completa de un individuo de Lama guanicoe macho de siete años o más que llevaba dos años muerto (dato corroborado con los puesteros) por causa desconoci$\mathrm{da}$, aproximadamente a 1,2 km del puesto. Dado que aún conservaba restos de cuero y tendones, se instaló la cámara en tres periodos consecutivos entre agosto del 2018 y mayo del 2019. El interés por esta carcasa era observar si, aun transcurrido tanto tiempo post mortem, algún carroñero se acercaba. Cabe aclarar que la posibilidad de acceder al campo y la duración de la batería de la cámara condicionaron el tiempo que duró cada monitoreo.

Para la confección de la base de datos y posterior análisis, se consideraron los siguientes atributos: fecha, hora, especie fotografiada, origen (autóctona/introducida), tasa de captura e interacción/no interacción con la carcasa (Forsyth et al., 2014; Cusimano, 2015). Este último criterio permitió diferenciar a los animales que solo están presentes de aquellos que efectivamente entran en contacto con la carcasa y constituyen potenciales agentes tafonómicos. El porcentaje de interacción con la carcasa fue calculado en base a la tasa de captura de cada taxón por monitoreo; estos fueron clasificados como bajos (1-30\%), moderados $(30-60 \%)$ y altos $(60-100 \%)$. La tasa de captura permite estimar de manera sencilla la riqueza de especies -no su abundancia relativa-, es decir, la cantidad de registros de individuos por especie durante el periodo de muestreo (Chávez et al., 2013).

Finalmente, durante cada monitoreo se realizaron observaciones preliminares sobre las modificaciones tafonómicas producidas en las carcasas. Para esto, se midió el área de dispersión de los huesos al final de cada monitoreo y se relevó la presencia de tejidos blandos, completitud, articulación entre elementos, enterramiento (Behrensmeyer y Dechantz Boas, 1988); y variables tafonómicas como meteorización, marcas de carnívoros, tinción por manganeso y pisoteo (Behrensmeyer, 1978; Haynes, 1980; Binford, 1981; Cusimano, 2015; Fernández-Jalvo y Andrews, 2016). Estas observaciones fueron realizadas in situ y no serán presentadas

\begin{tabular}{|c|c|c|c|c|c|c|}
\hline Monitoreo & Carcasas & $\begin{array}{l}\text { Fecha/ causa } \\
\text { de muerte }\end{array}$ & $\begin{array}{l}\text { Ambiente } \\
\text { inmediato }\end{array}$ & $\begin{array}{c}\text { Periodo } \\
\text { monitoreado }\end{array}$ & $\begin{array}{c}\text { Días } \\
\text { trampa }\end{array}$ & $\begin{array}{c}\mathrm{N} \\
\text { fotografías }\end{array}$ \\
\hline \multirow{2}{*}{1} & \multirow{2}{*}{$\begin{array}{l}\text { Bos taurus } \\
5 \text { hembras } \\
\text { adultas } \\
\text { Completas }\end{array}$} & \multirow{2}{*}{$\begin{array}{c}\text { 28/11/2017 sa- } \\
\text { crificadas por } \\
\text { enfermedad }\end{array}$} & \multirow{2}{*}{$\begin{array}{c}\text { Planicie con escasa } \\
\text { vegetación lindante } \\
\text { con una represa para } \\
\text { ganado }\end{array}$} & $\begin{array}{l}28 / 11 / 2017 \\
14 / 12 / 2017\end{array}$ & 16 & 819 \\
\hline & & & & Subtotal & 16 & 819 \\
\hline \multirow{3}{*}{2} & \multirow{3}{*}{$\begin{array}{l}\text { Lama guanicoe } \\
1 \text { hembra } \\
\text { juvenil } \\
\text { Completa }\end{array}$} & \multirow{3}{*}{$\begin{array}{c}\text { 20/05/2018 } \\
\text { atacada por } \\
\text { perros }\end{array}$} & \multirow{3}{*}{$\begin{array}{c}\text { Planicie con escasa } \\
\text { vegetación, aledaña } \\
\text { al puesto }\end{array}$} & $\begin{array}{l}31 / 5 / 2018 \\
23 / 6 / 2018\end{array}$ & 24 & 597 \\
\hline & & & & $\begin{array}{l}25 / 6 / 2018 \\
17 / 8 / 2018\end{array}$ & 53 & 203 \\
\hline & & & & Subtotal & 77 & 800 \\
\hline \multirow{4}{*}{3} & \multirow{4}{*}{$\begin{array}{c}\text { Lama guanicoe } \\
1 \text { macho adulto } \\
\text { Carpos, tarsos, } \\
\text { autopodios y } \\
\text { vértebras cau- } \\
\text { dales ausentes/ } \\
\text { Restos de cuero } \\
\text { y tendones }\end{array}$} & \multirow{4}{*}{ са. 06/2016 } & \multirow{4}{*}{$\begin{array}{c}\text { Planicie con abun- } \\
\text { dante vegetación } \\
\text { arbustiva }\end{array}$} & $\begin{array}{c}18 / 8 / 2018 \\
1 / 9 / 2018\end{array}$ & 14 & 10 \\
\hline & & & & $\begin{array}{c}\text { 10/10/2018 } \\
7 / 11 / 2018\end{array}$ & 29 & 82 \\
\hline & & & & $\begin{array}{l}27 / 3 / 2019 \\
19 / 5 / 2019\end{array}$ & 58 & 25 \\
\hline & & & & Subtotal & 101 & 117 \\
\hline & & & & Total & 194 & 1736 \\
\hline
\end{tabular}


pormenorizadamente, pero nos permitieron asociar los resultados del registro fotográfico con algunas de las alteraciones observadas en las carcasas.

\section{RESULTADOS}

La tasa de captura total de los monitoreos fue de 5869 (para acceder a la base de datos, ver Material suplementario), entre los que se incluyen aves $(77,15 \%)$ y mamíferos $(22,85 \%$ ) (Tabla 2$)$. El $89,86 \%$ de los animales registrados corresponde a especies autóctonas, mientras que el 9,83\% corresponde a especies introducidas, y el $0,31 \%$ restante, a animales no identificados. Esta tendencia se repitió en todos los monitoreos, aunque la diferencia entre autóctonos e introducidos se reduce en
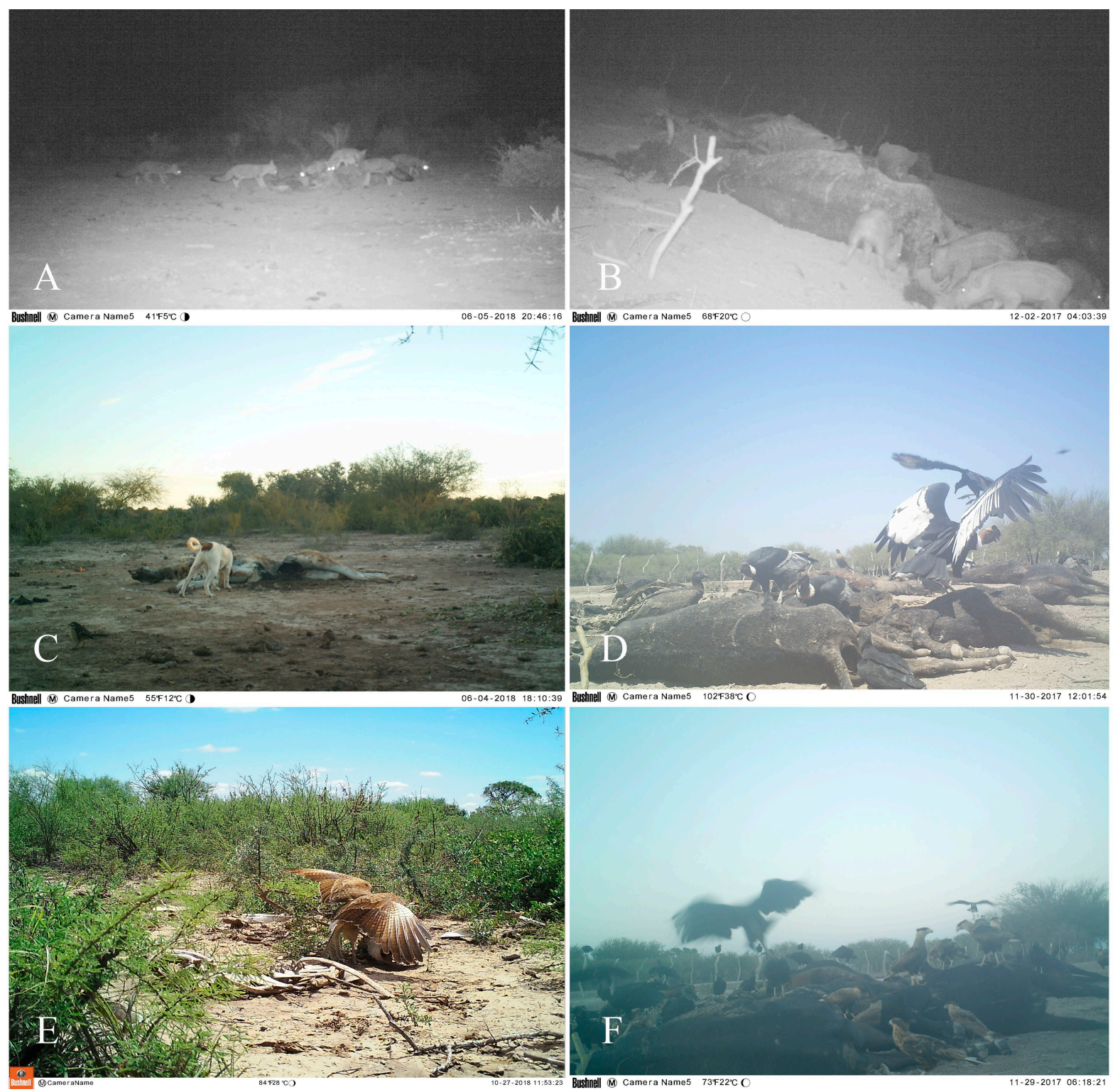

Figura 2. Principales agentes identificados: A) Lycalopex griseus; B) Sus scrofa, individuos juveniles; C) Canis lupus familiaris: D) Vultur gryphus y Coragyps atratus; E) Milvago chimango; F) Caracara plancus.

los monitoreos 2 y 3 , en los que se observa una tasa relativamente mayor de animales introducidos $(\approx 25 \%)$. En cuanto al porcentaje de interacción, el monitoreo 1 presenta una amplia mayoría de animales interactuando con la carcasa $(98,97 \%)$, seguido por el monitoreo 2, con un $83,49 \%$, y el monitoreo 3 , con un $57,36 \%$ (Tabla 2 ).

\section{Monitoreo 1}

Como se observa en la Tabla 2, el registro fotográfico del monitoreo 1 está compuesto en un 93\% por aves. Entre estas, las principales especies registradas fueron $C$. atratus, C. plancus y $V$. gryphus (Figura 2D), todas con un porcentaje de interacción superior al $99 \%$. El restante $7 \%$ de los animales registrados corresponde a mamíferos (Tabla 2), entre
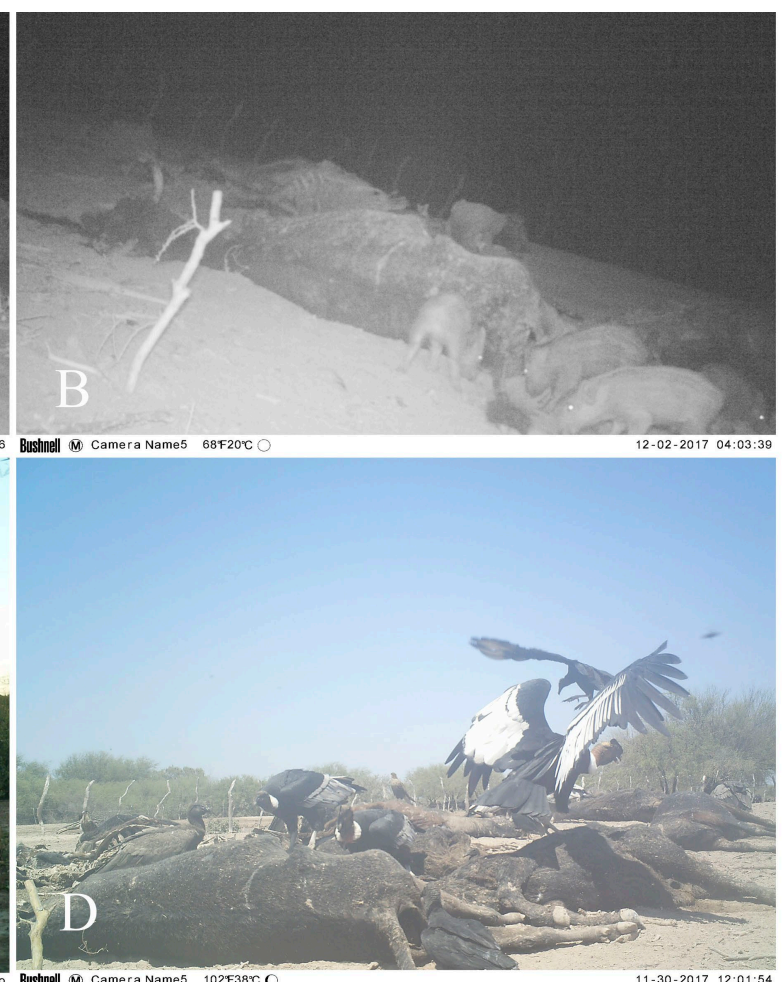


\begin{tabular}{|c|c|c|c|c|c|c|c|c|c|c|c|}
\hline \multirow[t]{2}{*}{ Taxón fotografiado } & \multicolumn{3}{|c|}{ Monitoreo 1} & \multicolumn{3}{|c|}{ Monitoreo 2} & \multicolumn{3}{|c|}{ Monitoreo 3} & \multirow[t]{2}{*}{ Total F } & \multirow{2}{*}{$\begin{array}{c}\text { Total } \\
\text { TC }\end{array}$} \\
\hline & $\mathbf{F}$ & TC & $\%$ I & $\mathbf{F}$ & TC & $\%$ I & $\mathbf{F}$ & TC & $\%$ I & & \\
\hline AVES & 1267 & 4348 & 99,52 & 64 & 86 & 50 & 88 & 94 & 62,77 & 1419 & 4528 \\
\hline Accipitriformes & 670 & 2843 & 99,61 & - & - & - & - & - & - & 670 & 2843 \\
\hline Cathartes aura & 10 & 14 & 100 & - & - & - & - & - & - & 10 & 14 \\
\hline Coragyps atratus & 502 & 2116 & 99,62 & - & - & - & - & - & - & 502 & 2116 \\
\hline Vultur gryphus & 149 & 697 & 99,57 & - & - & - & - & - & - & 149 & 697 \\
\hline No identificado & 9 & 16 & 100 & - & - & - & - & - & - & 9 & 16 \\
\hline Cariamiformes & - & - & - & 19 & 26 & 69,23 & 7 & 7 & 14,28 & 26 & 33 \\
\hline Chunga burmeisteri & - & - & - & 19 & 26 & 69,23 & 7 & 7 & 14,28 & 26 & 33 \\
\hline Columbiformes & 2 & 3 & 0 & 6 & 14 & 0 & 2 & 3 & 0 & 10 & 20 \\
\hline Patagioenas maculosa & 2 & 3 & 0 & 6 & 14 & 0 & 2 & 3 & 0 & 10 & 20 \\
\hline Estrutioniformes & - & - & - & 12 & 12 & 0 & 5 & 5 & 0 & 17 & 17 \\
\hline Rhea americana & - & - & - & 12 & 12 & 0 & 5 & 5 & 0 & 17 & 17 \\
\hline Falconiformes & 545 & 1415 & 99,86 & 20 & 26 & 80,76 & 56 & 60 & 86,67 & 621 & 1501 \\
\hline Caracara plancus & 518 & 1384 & 99,93 & 20 & 26 & 80,76 & 2 & 2 & 100 & 540 & 1412 \\
\hline Milvago chimango & 22 & 25 & 100 & - & - & - & 54 & 58 & 86,2 & 76 & 83 \\
\hline No identificado & 5 & 6 & 83,33 & - & - & - & - & - & - & 5 & 6 \\
\hline Passeriformes & 6 & 6 & 1,66 & 1 & 1 & 0 & 4 & 4 & 75 & 11 & 11 \\
\hline Coryphistera alaudina & 1 & 1 & 0 & - & - & - & 2 & 2 & 100 & 3 & 3 \\
\hline Machetornis rixosa & 4 & 4 & 0 & - & - & - & - & - & - & 4 & 4 \\
\hline Stigmatura budytoides & - & - & - & 1 & 1 & 0 & - & - & - & 1 & 1 \\
\hline Tyrannus savana & - & - & - & - & - & - & 2 & 2 & 50 & 2 & 2 \\
\hline No identificado & 1 & 1 & 100 & - & - & - & - & - & - & 1 & 1 \\
\hline Tinamiformes & - & - & - & 1 & 2 & 0 & 7 & 8 & 0 & 8 & 10 \\
\hline Eudromia elegans & - & - & - & 1 & 2 & 0 & 7 & 8 & 0 & 8 & 10 \\
\hline No identificado & 44 & 81 & 97,53 & 5 & 5 & 80 & 7 & 7 & 28,57 & 56 & 93 \\
\hline MAMMALIA & 278 & 315 & 91,43 & 740 & 992 & 86,39 & 28 & 34 & 44,12 & 1046 & 1341 \\
\hline Artiodactyla & 135 & 308 & 93,18 & 94 & 143 & 91,61 & 8 & 13 & 30,77 & 237 & 464 \\
\hline * Bos taurus & 20 & 26 & 19,23 & 12 & 16 & 25 & 3 & 6 & 66,66 & 35 & 48 \\
\hline Lama guanicoe & - & - & - & - & - & - & 1 & 1 & 0 & 1 & 1 \\
\hline * Sus scrofa & 115 & 282 & 100 & 82 & 127 & 100 & 1 & 3 & 0 & 198 & 412 \\
\hline No identificado & - & - & - & - & - & - & 3 & 3 & 0 & 3 & 3 \\
\hline Carnivora & 3 & 3 & 0 & 613 & 809 & 89,37 & 7 & 7 & 85,71 & 623 & 819 \\
\hline * Canis lupus familiaris & 3 & 3 & 0 & 105 & 125 & 67,2 & - & - & - & 108 & 128 \\
\hline Conepatus chinga & - & - & - & 1 & 1 & 0 & - & - & - & 1 & 1 \\
\hline Lycalopex griseus & - & - & - & 507 & 683 & 93,55 & 7 & 7 & 85,71 & 514 & 690 \\
\hline Cingulata & - & - & - & 4 & 4 & 0 & - & - & - & 4 & 4 \\
\hline Tolypeutes matacus & - & - & - & 4 & 4 & 0 & - & - & - & 4 & 4 \\
\hline Lagomorpha & - & - & - & 2 & 2 & 0 & - & - & - & 2 & 2 \\
\hline * Lepus europaeus & - & - & - & 2 & 2 & 0 & - & - & - & 2 & 2 \\
\hline Perissodactyla & - & - & - & 10 & 11 & 27,27 & 7 & 8 & 50 & 17 & 19 \\
\hline * Equus sp. & - & - & - & - & - & - & 3 & 3 & 33,33 & 3 & 3 \\
\hline * Equus africanus asinus & - & - & - & 3 & 4 & 50 & 2 & 3 & 66,66 & 5 & 7 \\
\hline * Equus asinus x caballus & - & - & - & 7 & 7 & 14,28 & 1 & 1 & 0 & 8 & 8 \\
\hline * Equus ferus caballus & - & - & - & - & - & - & 1 & 1 & 100 & 1 & 1 \\
\hline Rodentia & - & - & - & 15 & 21 & 0 & - & - & - & 15 & 21 \\
\hline Dolichotis patagonum & - & - & - & 1 & 1 & 0 & - & - & - & 1 & 1 \\
\hline Microcavia australis & - & - & - & 14 & 20 & 0 & - & - & - & 14 & 20 \\
\hline No identificado & 2 & 4 & 25 & 2 & 2 & 0 & 6 & 6 & 16,66 & 10 & 12 \\
\hline Total general & & 4663 & $\mathbf{9 8 , 9 7}$ & & 1078 & 83,49 & & 129 & 57,36 & & 5869 \\
\hline
\end{tabular}

$\mathrm{F}=$ fotografías en las que fue registrado un taxón; TC = tasa de captura ; \% I = porcentaje de interacción y * especies introducidas.

Tabla 2. Taxones registrados en cada uno de los monitoreos. 
los que destaca $S$. scrofa. Es interesante resaltar el registro de crías de $S$. scrofa alimentándose de las carcasas (Figura 2B), así como también de individuos juveniles de $V$. gryphus.

La única especie registrada en todos los periodos monitoreados fue C. plancus (Figura 2F), pero su tasa de captura fue mermando hacia el final del seguimiento (Figura 3). La presencia de C. atratus estuvo concentrada principalmente en la primera semana post mortem y cesó luego de 14 días, a diferencia de $V$. gryphus, cuya presencia se limitó a la primera semana (Figura 3). Se observó una superposición de la actividad de estas especies y del resto de las aves identificadas a lo largo del día. El patrón de actividad diario más constante fue el de $C$. atratus, cuyo pico de acción rondó entre las 6:00-12:00 h. De manera similar, C. plancus fue registrado durante todo el día, pero con mayores concentraciones entre las 6:00-18:00 h. En cambio, V. gryphus se concentró exclusivamente entre las 6:00-18:00 h.
18:00-6:00 h, con mayor tasa de captura a partir de las 0:00 h. (Figura 4). S. scrofa se presentó generalmente en parejas, con hasta cinco individuos actuando sincrónicamente. Por último, también se cuenta con registros de B. taurus y C. lupus familiaris, con porcentajes de interacción bajo y nulo, respectivamente (Tabla 2, Figura 3).

\section{Monitoreo 2}

En el segundo monitoreo, las proporciones de aves y mamíferos fueron inversas con respecto al monitoreo 1, con un 92\% de representación de mamíferos. Entre ellos, se relevó una intensa actividad carroñera de cánidos -autóctonos e introducidosy S. scrofa. L. griseus fue la especie con la tasa de captura más alta (Tabla 2), con un porcentaje de interacción superior al 90\%. Su tasa de captura disminuyó luego de las dos primeras semanas, pero fue registrado a lo largo de todo el monitoreo

(Figura 4). C. atratus y V. gryphus actúan en grupos de cinco individuos en promedio, con máximos de hasta 16-18 individuos en simultáneo, respectivamente. $C$. plancus se presenta principalmente en parejas o tríos, con un máximo de 13 individuos registrados simultáneamente. Por otra parte, cabe mencionar la presencia, si bien con una tasa de captura baja y nula interacción, de C. alaudina, M. rixosa y P. maculosa (Tabla 2, Figura 3).

Con respecto a la actividad de mamíferos, se destaca la actividad de $S$. scrofa a lo largo del monitoreo, que fue decreciendo hacia los últimos días (Figura 3). La especie fue registrada entre las
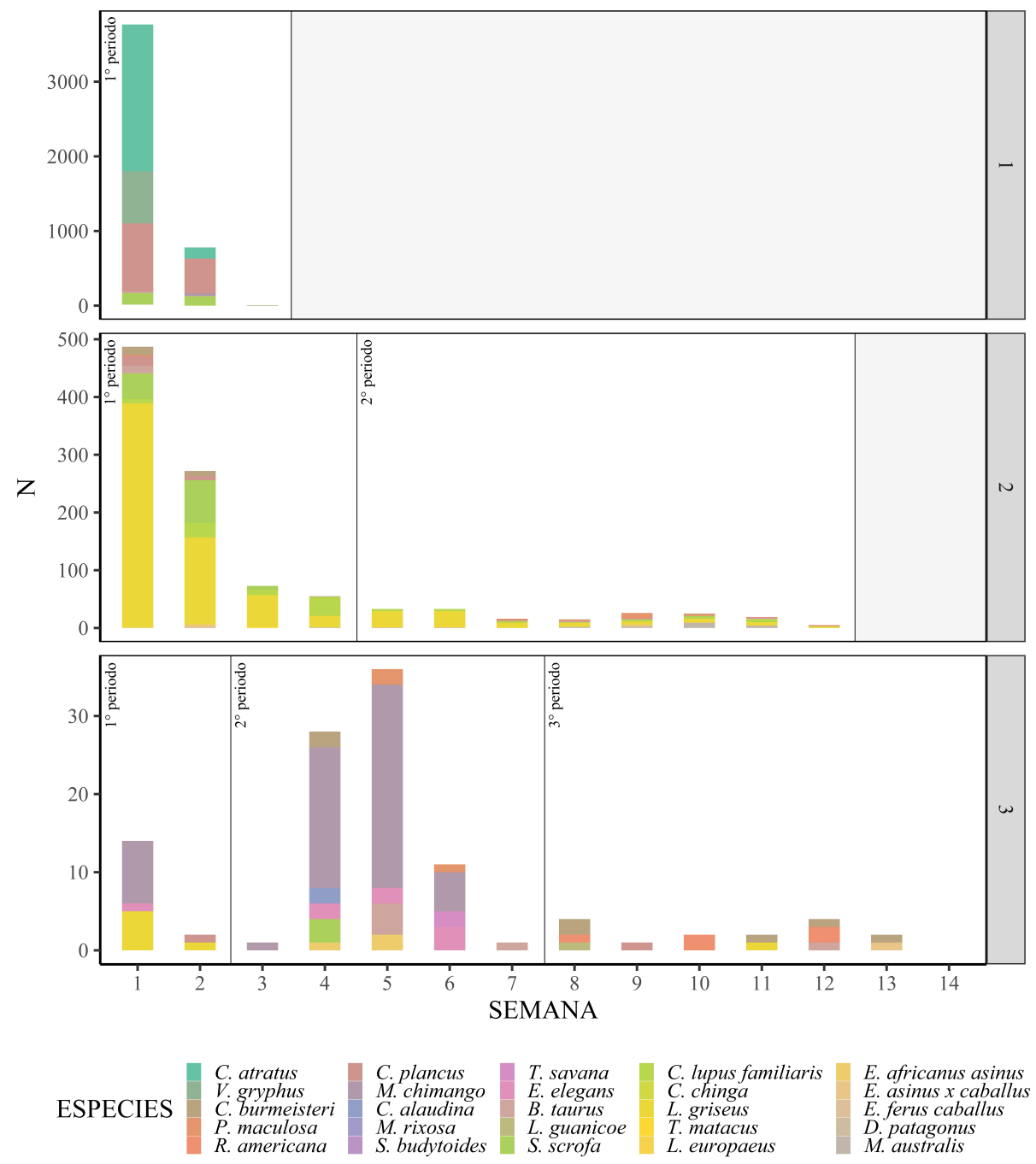

Figura 3. Tasa de captura por especie registrada semanalmente en el transcurso de los tres monitoreos realizados. 
(Figura 3). En general, se observó que actúan solos o en parejas, con máximos de hasta siete individuos alimentándose de la carcasa simultáneamente.

La segunda especie con mayor tasa de captura fue S. scrofa, con un $100 \%$ de interacción con la carcasa (Tabla 2). Por otra parte, la tasa de captura de C. lupus familiaris fue similar, pero presentó un porcentaje de interacción moderado (Tabla 2). Estos aparecieron esporádicamente durante el monitoreo, con un pico de observaciones a comienzos del segundo periodo (Figura 3). Ambas especies fueron registradas tanto en solitario como en parejas y tríos. En cuanto al patrón de actividad diario de L. griseus, C. lupus familiaris y S. scrofa, las tres especies fueron registradas principalmente durante la noche (Figura 4).

Un aspecto destacable de este monitoreo fue el registro de mamíferos de los órdenes Rodentia, Cingulata y Lagomorpha, que no fueron identificados en ninguno de los otros monitoreos (Tabla 2). Asimismo, se registró la presencia de bóvidos y équidos de manera esporádica en los alrededores de la carcasa (Figura 3), con porcentajes de interacción que van de moderados a bajos (Tabla 2).

Con respecto a las aves, que componen el restante $8 \%$ de animales identificados, se destaca la presencia de C. burmeisteri y C. plancus, ambos con una tasa de captura de 26, pero con mayor interacción por parte del falconiforme (Tabla 2). Por último, fueron identificados ejemplares de $P$. maculosa y $R$. americana, con baja tasa de captura y nula interacción (Tabla 2).

\section{Monitoreo 3}

M. chimango (Figura 2E) fue la especie con la tasa de captura más alta durante este monitoreo. De hecho, el 45\% de los registros del monitoreo 3 corresponde a esta especie, que presentó un porcentaje de interacción con la carcasa cercano al 90\% (Tabla 2). No obstante, la especie no fue registrada durante el tercer periodo monitoreado (Figura 3). Su patrón de actividad diario se concentró mayormente entre las 6:00-18:00 h. (Figura 4) y fue solitario, aunque ocasionalmente se registró en parejas. En cuanto al resto de las aves identificadas, las tasas de captura no superaron los ocho individuos. C. plancus y las paseriformes $C$. alaudina y $T$. savanna presentaron porcentajes de interacción moderados a altos, mientras que $C$. burmeisteri, $P$. maculosa y $R$. americana presentaron porcentajes de interacción bajos a nulos (Tabla 2).

En relación con los mamíferos, se destaca la presencia de $L$. griseus, con alto porcentaje de interacción, pero identificado esporádicamente a lo largo del monitoreo (Tabla 2, Figura 3). Esta especie fue registrada de manera solitaria entre las 0:0018:00 h. (Figura 4). Por otra parte, tanto bóvidos como équidos se encuentran bien representados en el registro. En cambio, S. scrofa y $L$. guanicoe fueron registrados por única vez, aunque sin interacción con la carcasa (Tabla 2, Figura 3).

\section{Observaciones preliminares sobre dispersión y modificaciones óseas}

Con respecto a las modificaciones óseas, la Figura 5 muestra algunos de los registros realizados
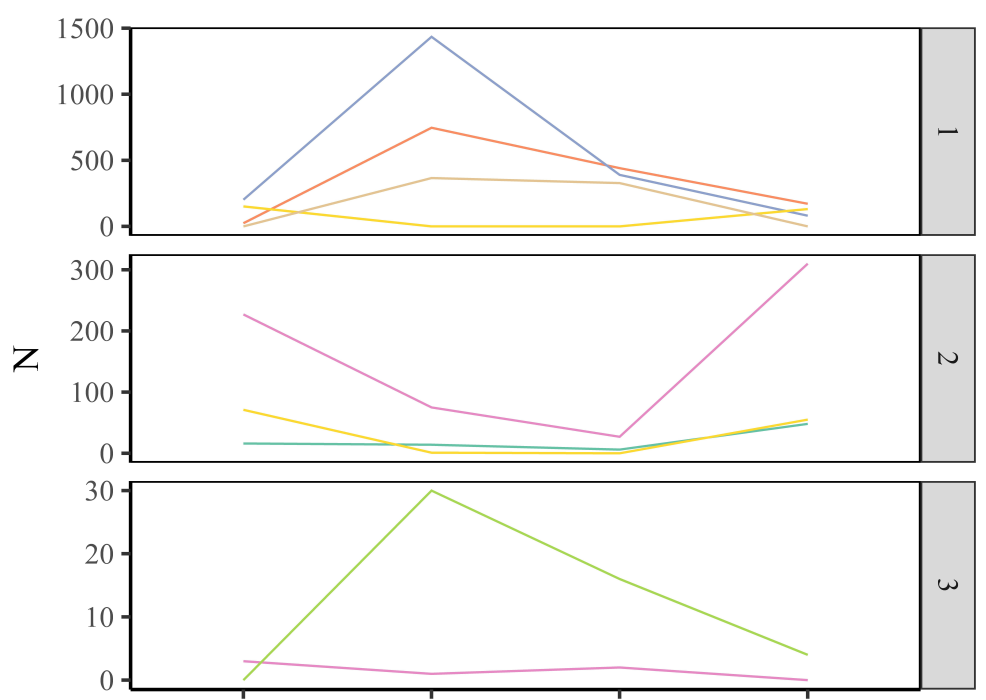

0:00 a 6:00 hs. $6: 00$ a 12:00 hs 12:00 a 18:00 hs 18:00 a 0:00 hs Rango horario
Principales especies identificadas

- Canis lupus familiaris

- Caracara plancus

- Coragyps atratus

- Lycalopex griseus

- Milvago chimango

- Sus scrofa

- Vultur gryphus

Figura 4. Patrón de actividad diario de las principales especies identificadas interactuando con la carcasa por monitoreo. 
in situ. Nuestras observaciones apuntan a que $S$. scrofa (Figura 5A) y cánidos fueron las especies que mayor impacto tuvieron en la desarticulación y dispersión de las carcasas. En el monitoreo 1 se registraron individuos de S. scrofa moviendo carcasas de vacas prácticamente completas por varios metros $y$, finalizado el monitoreo, los huesos se hallaron dispersos en un área de $40 \mathrm{~m}^{2}$. En el monitoreo 2, la carcasa estuvo sujeta a un acelerado proceso de descarne y desarticulación producto de la acción combinada de S. scrofa, C. lupus familiaris y L. griseus. Al finalizar el primer periodo del monitoreo 2, la carcasa solo conservaba partes del cuero y restos de tendones, y los elementos se encontraron dispersos en un área de $260 \mathrm{~m}^{2}$. En la Figura 5B se aprecian el cráneo, con una fractura en la porción nasal, y las primeras cervicales, hallados articulados a $17 \mathrm{~m}$ de su posición inicial. Las costillas fueron los elementos más afectados por la acción de estas especies; esto se evidenció en una gran cantidad de modificaciones (Figura 5C), fragmentación y destrucción de especímenes.

La acción del $L$. griseus es interesante en tanto único mamífero autóctono registrado consumiendo las carcasas y, adicionalmente, por su interacción con la carcasa ya esqueletizada del monitoreo 3 (Figura 5D). En este último, perforaciones y arrastres fueron registrados en las epífisis de húmeros y fémures (Figura 5E). Cabe destacar que, tras un periodo de fuertes lluvias entre marzo y mayo de 2019, los escasos tejidos blandos remanentes fueron rehidratados y se observaron nuevas marcas de carnívoros en los huesos largos. La dispersión fue menor comparada con el monitoreo 2 (ca. $40 \mathrm{~m}^{2}$ ).

Ungulados de gran porte, como $B$. taurus, Equus sp. y L. guanicoe, fueron fotografiados en los tres monitoreos $y$, aunque en la mayoría de los casos no interactuaron con la carcasa, son los responsables de fracturas y marcas lineales por pisoteo. La carcasa del monitoreo 3, ya esqueletizada, fue la más afectada por este proceso. Se observaron fracturas en costillas, escápula y procesos vertebrales durante los primeros dos periodos, mientras que en el tercero se sumaron fracturas espiraladas en la tibia izquierda y el fémur derecho.
Con respecto a las aves, se considera que en los monitoreos 1 y 2 su potencial para dejar marcas en los huesos fue bajo dado que, en el primer caso, las carcasas aún se encontraban con gran parte de los tejidos blandos y estas se concentraron en el consumo de ojos y vísceras; y en el segundo, la intensa actividad de mamíferos habría limitado su actividad. En el monitoreo 3, en cambio, se registró el movimiento del cráneo por parte de $C$. alaudina, a pesar de su pequeño porte, y $M$. chimango fue fotografiado picoteando el cráneo y los huesos del tórax (Material suplementario). No obstante, la dispersión ocasionada por estas especies fue mínima y no se observaron trazas identificables en los huesos.

\section{DISCUSIÓN}

La relación entre humanos y animales carroñeros ha sido escasamente explorada en la literatura zooarqueológica de la provincia de Córdoba. En efecto, una revisión bibliográfica de los estudios zooarqueológicos regionales publicados en los últimos años muestra que la información sobre carnívoros y/o carroñeros suele limitarse a su identificación taxonómica -marginal- dentro de los conjuntos y, ocasionalmente, al reporte de marcas de carnívoros como variable tafonómica genérica (i.e., sin distinción entre tipos de marcas o niveles de daño) en la sección de resultados, sin mayores discusiones

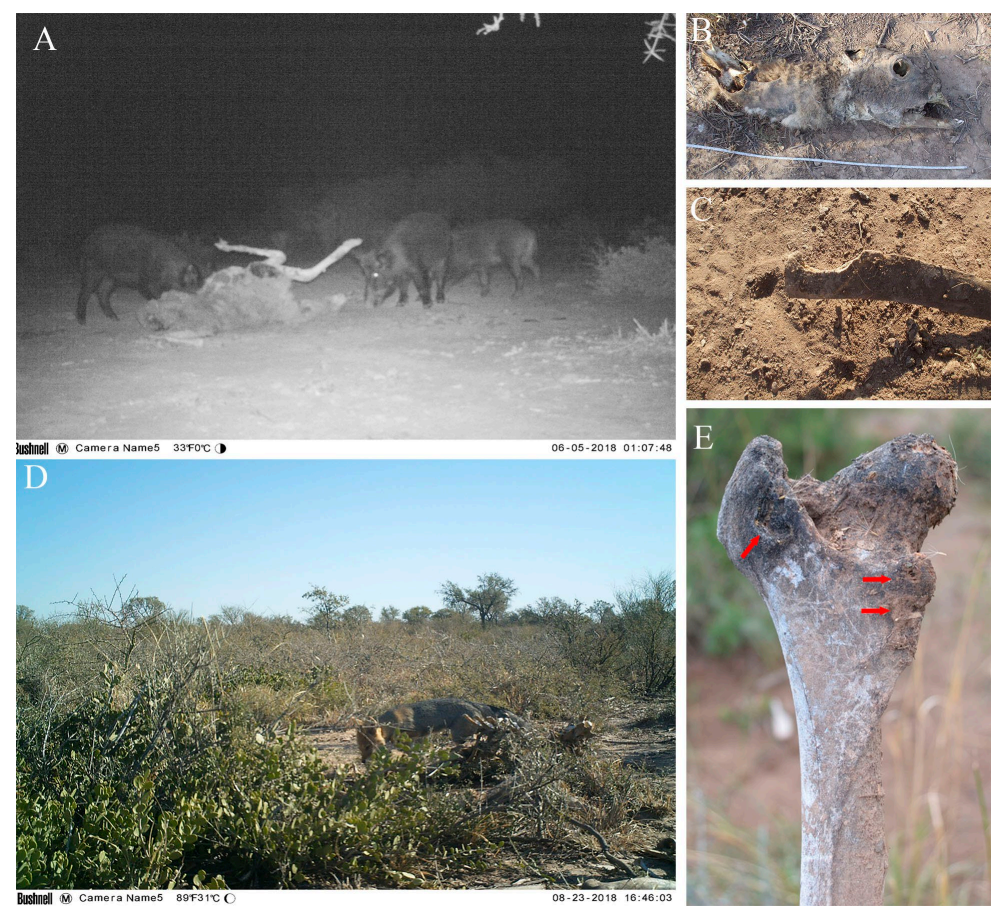

Figura 5. Monitoreo 2: A) S. scrofa moviendo la carcasa; B) Cráneo y cervicales de $L$. guanicoe desarticulados; C) Mordisqueo en la hoja de una costilla. Monitoreo 3: D) L. griseus; E) Daño asociado a la acción de L. griseus. 
sobre sus implicancias (e.g., Rivero et al., 2010; Medina, 2014; Costa, 2016). No obstante, nuestros resultados evidencian que las especies involucradas en el reciclaje de carcasas de ungulados en el área de estudio son numerosas y exceden a las habitualmente consideradas en estudios zooarqueológicos regionales, aunque no necesariamente dejan trazas tafonómicas concretas.

De acuerdo con nuestro registro, las accipitriformes son las primeras en acceder a las carcasas y presentan las mayores tasas de captura y porcentajes de interés. El gran porte y la morfología del pico de $V$. gryphus le permiten abrir las regiones abdominales de los cadáveres, y así dejan expuestas las vísceras, que luego quedan disponibles para aves menores (Wallace y Temple, 1987), como fue observado en el monitoreo 1 (Material suplementario). Estudios tafonómicos forenses coinciden en que su actividad puede acelerar el proceso de esqueletización en humanos (Ubelaker y DeGaglia, 2020), algo que también fue observado en ovejas y liebres en Norpatagonia (Ballejo, 2016). Sin embargo, la información sobre las trazas generadas por accipitriformes es disímil. Reeves (2009) señala que los cóndores producen rasguños en los huesos humanos, mientras que Spradley, Hamilton y Giordano (2012) no registraron trazas, pero sí una considerable dispersión de elementos. Ninguno de estos procesos fue observado en nuestros monitoreos. Teniendo en cuenta la superposición con otros procesos tafonómicos, como la meteorización, y el mayor porte de las carcasas monitoreadas en relación con las de estudios previos, no sería esperable hallar trazas de accipitriformes en el registro zooarqueológico de ungulados del noroeste de Córdoba. Esta premisa aplicaría, por consiguiente, a las aves de menor porte identificadas en el registro fotográfico de los tres monitoreos.

En cuanto a los mamíferos, la mayor tasa de captura fue de L. griseus, un carroñero oportunista de hábitos nocturnos que circunstancialmente puede estar activo durante el día (Nowak y Paradiso, 1983) (Figura 4). De acuerdo con Borrero (2007), la acción de zorros tiende a ser intensa en los primeros meses tras la muerte de un animal y luego se vuelve más intermitente. Esta observación coincide con lo registrado en los monitoreos 2 y 3 , pero no explica la ausencia de esta especie en el monitoreo 1. A modo de hipótesis, consideramos que la actividad constante de aves carroñeras y S. scrofa podría haber desalentado el acercamiento de un carroñero oportunista y de menor porte como L. griseus, aunque otros factores pueden haber influido. Al respecto, el registro obtenido en el monitoreo 2 muestra que estos cánidos se retiran ante la llegada de especies de mayor porte, como $S$. scrofa o C. lupus familiaris (Material suplementario).

En relación con el potencial tafonómico de $L$. griseus, estudios de corte naturalista sobre la acción de la especie en carcasas de ungulados coinciden en que el daño causado por estos cánidos es ligero (Borrero, 1990; Nasti, 2000), y las trazas más comúnmente impresas en los huesos son hoyuelos, perforaciones y arrastres finos (Mondini, 2018), en concordancia con nuestras observaciones preliminares (Figura 5E). Asimismo, Mondini (2018) señala que, aun cuando las carcasas han sido despojadas de la mayor parte de sus tejidos blandos, los zorros continúan acercándose para alimentarse de los huesos y el cuero, tal como fue observado en el monitoreo 3 (Figura 5D).

En base a un estudio desarrollado en la Isla Grande de Tierra del Fuego (Argentina), Borrero (2007) señala que $L$. griseus interviene en la desarticulación y dispersión de los restos de L. guanicoe, pero no acelera el proceso en sí. En caso de actuar más de un zorro sobre la carcasa, estos tienden a transportar elementos o porciones esqueletales a distancias cortas (Borrero, 1990, 2007). En relación con esto último, en el monitoreo 3 -donde la acción dispersora del zorro pudo ser aislada de la del jabalí-, el radioulna izquierdo fue hallado a $14 \mathrm{~m}$ al noroeste de la carcasa, y las cervicales (II-III), aún con cuero, a unos $10 \mathrm{~m}$ al sureste. No obstante, en este caso, la especie fue registrada de manera solitaria, lo que no coincidiría plenamente con lo planteado por el autor.

Por otra parte, S. scrofa también jugó un papel importante en el carroñeo de las carcasas: presentó una de las mayores tasas de captura y porcentajes de interés dentro del grupo de mamíferos. Las especies exóticas invasoras, como S. scrofa, son oportunistas y fácilmente adaptables a nuevos hábitats, lo que les permite aumentar rápidamente sus poblaciones; resultan, además, la principal causa de extinción, retracción y reestructuración de las poblaciones biológicas nativas (Novillo y Ojeda, 2008). En esta línea, es interesante destacar la ausencia de registros de las dos especies de suidos autóctonos presentes en el área: Catagonus wagneri y Pecari tajacu, especializadas en el consumo de cactáceas, a las que se les han atribuido hábitos carroñeros (Taber et al., 1994; Salazar, 2013). 
En las etapas iniciales del proceso de descomposición (monitoreos 1 y 2), fue llamativo el potencial de dispersión de los individuos de S. scrofa, capaces de mover varios metros las carcasas enteras y grandes porciones anatómicas, así como su rol en la desarticulación (Material suplementario). En relación con esto, Domínguez-Solera y DomínguezRodrigo (2009) realizaron un estudio tafonómico sobre las modificaciones producidas por suidos domésticos y salvajes de origen europeo en huesos largos de diversas especies. En él registraron que estos mamíferos son capaces de modificar los huesos de manera similar a los grandes carnívoros como hienas y perros, aunque su capacidad para modificar huesos de animales mayores a $100 \mathrm{~kg}$ es más limitada. Además, las marcas generadas por sus dientes poseen una distribución y características distinguibles de aquellas producidas por cánidos (Greenfield, 1988) como, por ejemplo, arrastres longitudinales en las escápulas; información a tener en cuenta para el futuro análisis de los elementos esqueletales.

A diferencia de L. griseus, a C. lupus familiaris y $S$. scrofa se les atribuye una mayor capacidad de modificación y destrucción ósea (Marean y Spencer, 1991; Domínguez-Solera y Domínguez-Rodrigo, 2009), aunque la magnitud de estos cambios puede ser altamente variable. Particularmente en el monitoreo 2, la superposición de la acción de S. scrofa, C. Iupus familiaris y L. griseus vuelve ambigua la distinción en campo de las modificaciones generadas por cada taxón. No obstante, podemos afirmar que cuando estos tres mamíferos coinciden en el carroñeo de una carcasa, se produce un aumento de la dispersión y destrucción ósea, fenómeno que ha sido observado en otros ambientes (e.g., Faith, Marean y Behrensmeyer, 2007; Arilla et al., 2020; Wadley 2020). En efecto, Haynes (1982) ha señalado que entre los factores a considerar a la hora de evaluar el grado de destrucción ósea se cuentan la competencia entre carroñeros y la cantidad de carcasas disponibles, variables que deberán ser incorporadas en futuros análisis.

Por último, al poner el foco en las especies con mayor interés en las carcasas, no desestimamos las alteraciones no deliberadas que otras especies puedan realizar sobre ellas. Nuestros monitoreos muestran que ungulados autóctonos como L. guanicoe e introducidos como Equus sp., B. taurus y S. scrofa circulan por el área y generan marcas de pisoteo sobre las osamentas. De hecho, en el monitoreo 3 se observaron fracturas helicoidales en huesos largos que fueron atribuidas al pisoteo de huesos meteorizados (Haynes, 1982), dado que no se registraron carnívoros capaces de generar fracturas semejantes. En tanto, el pisoteo es un proceso tafonómico que merece atención en la región, puesto que puede operar durante largo tiempo luego de que los carroñeros han perdido interés en los huesos (Borrero, 1990; Cusimano, 2015).

En relación con la arqueología regional, consideramos que las bajas o nulas frecuencias reportadas de especímenes con marcas de carnívoros en los estudios zooarqueológicos del noroeste de la provincia de Córdoba (Rivero et al., 2010; Medina, 2014; Costa, 2016) han Ilevado a desestimar el rol de animales carroñeros en la formación de los conjuntos zooarqueológicos. Sin embargo, sobre la base de nuestros resultados y conscientes de la naturaleza promediada del registro arqueológico, creemos que el Ilamado "efecto dilución" (sensu Mondini, 2003) podría afectar la posibilidad analítica de distinguir las estructuras de conjuntos pequeños generados por animales carroñeros dentro de depósitos arqueológicos más grandes donde la actividad humana ha sido la principal acumuladora e invisibilizar así el rol de agentes no-humanos. En este sentido, identificar a los potenciales agentes modificadores del registro zooarqueológico a nivel local y comenzar a reconocer sus consecuencias es clave para discernir entre la actividad humana y la no-humana.

\section{CONSIDERACIONES FINALES}

Se presentaron tres monitoreos actualísticos con cámaras trampa sobre carcasas de grandes ungulados en el extremo sur de las Salinas Grandes de Córdoba, Argentina. La diversidad de carroñeros identificados sugiere que las modificaciones observadas en el registro zooarqueológico de la región pueden haber sido producidas por una variedad de animales que excede a los habitualmente considerados. Las especies con mayor potencial de generar modificaciones tafonómicas fueron $L$. griseus, $S$. scrofa y C. lupus familiaris. La actividad de L. griseus resalta por haber sido registrada tanto en las etapas iniciales de descomposición como en una carcasa esqueletizada. El daño generado por esta especie es ligero, lo que puede dificultar su reconocimiento en contextos arqueológicos. S. scrofa y C. lupus familiaris se presentaron en las etapas inmediatamente post mortem y fueron los principales responsables de la dispersión y destrucción ósea. Consideramos 
que, a pesar de su naturaleza exótica, el registro de la agencia tafonómica de estas especies es valioso para contextos arqueológicos históricos. Entre las aves, se destacó el registro de accipitriformes y falconiformes consumiendo las carcasas, pero su potencial para generar trazas detectables en el registro arqueológico es incierto. Por último, se registró pisoteo por parte de grandes ungulados, un proceso que a futuro debería evaluarse considerando otros factores como el sustrato y la topografía para comprender mejor los patrones de fractura y los procesos de migración vertical en el área.

Como agenda a futuro, pretendemos presentar resultados más detallados acerca de los procesos de desarticulación, dispersión y modificación ósea, y ampliar la cantidad de monitoreos para evaluar la influencia de variables como el ambiente de depositación, la estacionalidad de las muertes y la proximidad a la presencia humana. Esto contribuirá a ampliar nuestro conocimiento sobre los agentes tafonómicos locales, así como sobre la dinámica ecosistémica actual y, en última instancia, enriquecerá nuestras interpretaciones sobre el registro zooarqueológico local.

\section{Agradecimientos}

Este trabajo fue financiado por SeCyT de la Universidad Nacional de Córdoba (FORMAR, Res. 411/18), Ministerio de Ciencia y Tecnología de Córdoba (MincyT, GRFT Res. 77/19) y por el CONICET. Agradecemos a propietarios y trabajadores de La Providencia por permitirnos realizar este trabajo. Los comentarios de tres revisores anónimos enriquecieron en gran medida este trabajo; estamos agradecidos por sus aportes. Cualquier error persistente es nuestra responsabilidad.

\section{REFERENCIAS CITADAS}

Arilla, M., Rosell, J., y Blasco, R. (2020). A neotaphonomic approach to human campsites modified by carnivores. Scientific Reports Nature Research, 10(1), 6659. https://doi.org/10.1038/s41598-020-63431-8

Ballejo, F. (2016). Ecología trófica y tafonomía del jote cabeza negra, Coragyps atratus (Cathartidae) y su comparación con otros Cathartidae en el noroeste de la Patagonia (tesis doctoral). Universidad Nacional de La Plata, La Plata, Argentina.
Behrensmeyer, A. K. (1978). Taphonomic and ecologic information from bone weathering. Paleobiology, 4(2), 150-162. https://doi.org/10.1017/S0094837300005820

Behrensmeyer, A. K., y Dechant Boaz, E. (1988 [1980]) The recent bones of Amboseli Park, Kenya in relation to East African paleoecology. En Fossils in the making. Vertebrate taphonomy and paleoecology (pp. 72-93), 2a ed. Midway.

Behrensmeyer, A. K., Kidwell, S. M., y Gastaldo, R. A. (2000). Taphonomy and paleobiology. Paleobiology, 26(sp4), 103-147.

Behrensmeyer, A. K., y Miller, J. H. (2012). Building links between ecology and paleontology using taphonomic studies of recent vertebrate communities. En J. Louys (Ed.), Paleontology in ecology and conservation (pp. 69-91). Springer Berlin Heidelberg. https://doi. org/10.1007/978-3-642-25038-5_5

Binford, L. R. (1981). Bones: Ancient men and modern myths. Academic Press.

Blumenschine, R. J. (1989). A landscape taphonomic model of the scale of prehistoric scavenging opportunities. Journal of Human Evolution, 18(4), 345-371. https://doi. org/10.1016/0047-2484(89)90036-5

Borrero, L. A. (1990). Taphonomy of guanaco bones in Tierra del Fuego. Quaternary Research, 34(3), 361-371. https://doi.org/10.1016/0033-5894(90)90047-O

Borrero, L. A. (2007). Longitudinal taphonomic studies in Tierra del Fuego, Argentina. En M. A. Gutiérrez, L. Miotti, G. Barrientos, G. Mengoni Goñalons y M. Salemme (Eds.), Taphonomy and zooarchaeology in Argentina (pp. 219-233). Archaeopress.

Carignano, C. A., Kröhling, D., Degiovanni, S., y Cioccale, M. A. (2014). Geomorfología. En Relatorio del XIX Congreso Geológico Argentino (pp. 747-821). Asociación Geológica Argentina.

Chávez, C., De La Torre, A., Bárcenas, H., Medellín, R. A., Zarza, H., y Ceballos, G. (2013). Manual de fototrampeo para estudio de fauna silvestre. El jaguar en México como estudio de caso. Alianza WWF-Telcel, Universidad Nacional Autónoma de México.

Chen, M., Tewes, M. E., Pei, K. J., y Grassman Jr, L. I. (2009). Activity patterns and habitat use of sympatric small carnivores in southern Taiwan. Mammalia, 73(1), 20-26.

Costa, T. (2016). Una aproximación zooarqueológica a las prácticas de los cazadores-recolectores en el Alero Deodoro Roca (ca. 2970 AP), Valle de Ongamira, Córdoba. Revista del Museo de Antropología, 9(2), 93104. https://doi.org/10.31048/1852.4826.v9.n2.15326 
Costa, T., y Barri, F. (2018). Lama guanicoe remains from the Chaco ecoregion (Córdoba, Argentina): An osteological approach to the characterization of a relict wild population. Plos ONE, 13(4), e0194727. https:// doi.org/10.1371/journal.pone.0194727

Cusimano, D. A. (2015). Trampling modification. An actualistic study in taphonomy (Master of Arts in Anthropology). California State University, East Bay.

Domínguez-Solera, S. D., y Domínguez-Rodrigo, M. (2009). A taphonomic study of bone modification and of toothmark patterns on long limb bone portions by suids. International Journal of Osteoarchaeology, 19(3), 345363. https://doi.org/10.1002/oa.987

Erlandson, J. M., y Moss, M. L. (2001). Shellfish feeders, carrion eaters, and the archaeology of aquatic adaptations. American Antiquity, 66(3), 413-432.

Faith, J. T., Marean, C. W. y Behrensmeyer, A. K. (2007). Carnivore competition, bone destruction, and bone density. Journal of Archaeological Science, 34(12), 2025-2034. https://doi.org/10.1016/j.jas.2007.01.017

Fernandez-Jalvo, Y. y Andrews, P. (2016). Atlas of Taphonomic Identifications: 1001+ Images of Fossil and Recent Mammal Bone Modification. Springer Netherlands. https://doi.org/10.1007/978-94-017-7432-1

Forsyth, D. M., Woodford, L., Moloney, P. D., Hampton, J. O., Woolnough, A. P. y Tucker, M. (2014). How does a carnivore guild utilize a substantial but unpredictable anthropogenic food source? Scavenging on hunter-shot ungulate carcasses by wild dogs/dingoes, red foxes and feral cats in South-Eastern Australia revealed by camera traps. PloS ONE, 9(6). https://doi.org/10.1371/journal. pone.0097937

Greenfield, H. J. (1988). Special studies: Bone consumption by pigs in a contemporary Serbian village: Implications for the interpretation of prehistoric faunal assemblages. Journal of Field Archaeology, 15(4), 473-479.

Gutiérrez, M. A., Rafuse, D. J., Álvarez, M. C., Massigoge, A., González, M. E., Scheifler, N. A. y Kaufmann, C. A. (2018). Ten years of actualistic taphonomic research in the Pampas region of Argentina: Contributions to regional archaeology. Quaternary International, 492, 40-52. https://doi.org/10.1016/j.quaint.2017.09.025

Haynes, G. (1980). Evidence of carnivore gnawing on Pleistocene and recent mammalian bones. Paleobiology, 6(3), 341-351.

Haynes, G. (1982). Utilization and skeletal disturbances of North American prey carcasses. Arctic, 35(2), 266-281.

Marean, C. W. y Spencer, L. M. (1991). Impact of carnivore ravaging on zooarchaeological measures of element abundance. American Antiquity, 56(4), 645-658. https:// doi.org/10.2307/281542
Medina, M. E. (2014). Arqueofaunas y procesos de intensificación en el Período Prehispánico Tardío de las Sierras de Córdoba (Argentina): El sitio Arroyo Talainín 2. Comechingonia. Revista de Arqueología, 18(2), $117-$ 135. https://doi.org/10.37603/2250.7728.v18.n2.18157

Mondini, N. M. (2003). Formación del registro arqueofaunístico en abrigos rocosos de la Puna argentina. Tafonomía de carnívoros (tesis doctoral). Universidad de Buenos Aires, Buenos Aires, Argentina.

Mondini, M. (2018). Carnivore taphonomy in South America: A review of actualistic studies and their implications in the southern Neotropics. Historical Biology, 30(6), 774-785. https://doi.org/10.1080/089129 63.2017.1319831

Muñoz, A. E., Beltrami, E. y Osorio, C. (2017). Evaluación de presencia de mamíferos en predios frutícolas del norte chico y su variación a escala intra-predial. Sustentabilidad (es), 8(16), 46-60.

Nasti, A. (2000). Modification of vicuña carcasses in highaltitude deserts. Current Anthropology, 41(3), 279-283.

Novillo, A. y Ojeda, R. A. (2008). The exotic mammals of Argentina. Biological Invasions, 10(8). https://doi. org/10.1007/s10530-007-9208-8

Nowak, R. M. y Paradiso, J. L. (1983). Walker's mammals of the world: Vol. II. The Johns Hopkins University Press.

O'Connell, Jr. A. F., Nichols, J. D. y Karanth, U. K. (2011). Camera traps in animal ecology: methods and analyses. Springer.

Reeves, N. M. (2009). Taphonomic Effects of Vulture Scavenging. Journal of Forensic Sciences, 54(3), 523528. https://doi.org/10.1111/j.1556-4029.2009.01020.x

Ringuelet, R. A. (1961). Rasgos fundamentales de la zoogeografía argentina. Physis, 22(63), 151-170.

Rivero, D. E., Medina, M. E., Recalde, A. y Pastor, S. (2010). Variabilidad en la explotación de recursos faunísticos durante el Holoceno en las Sierras de Córdoba (Argentina): Una aproximación zooarqueológica. En M. A. Gutiérrez, M. De Nigris, P. M. Fernández, M. Giardina, A. F. Gil, A. D. Izeta, G. A. Neme y H. D. Yacobaccio (Eds.), Zooarqueología a principios del siglo XXI: aportes teóricos, metodológicos y casos de estudio (pp. 321-331). Ediciones del Espinillo.

Salazar, M. R. (2007). Dieta de los chanchos de monte: Taitetu (Tayassu tajacu) y Tropero (Tayassu pecari) en el territorio indígena Siriono, Beni, Bolivia. Revista Boliviana de Ecología y Conservación, 21, 43-56.

Spradley, M. K., Hamilton, M. D. y Giordano, A. (2012). Spatial patterning of vulture scavenged human remains. Forensic Science International, 219(1-3), 57-63. https:// doi.org/10.1016/j.forsciint.2011.11.030 
Taber, A. B., Doncaster, C. P., Neris, N. N. y Colman F. H. (1994). Ranging behavior and activity patterns of two sympatric peccaries, Catagonus wagneri \& Tayassu tajacu, in the Paraguayan Chaco. Mammalia, 58, 61-71.

Ubelaker, D. H. y DeGaglia, C. M. (2020). The impact of scavenging: Perspective from casework in forensic anthropology. Forensic Sciences Research, 5(1), 32-37. https://doi.org/10.1080/20961790.2019.1704473

Wadley, L. (2020). A camera trap record of scavengers at a kudu carcass: implications for archaeological bone accumulations. Transactions of the Royal Society of South Africa, 75(3), 245-257.

Wallace, M. P. y Temple, S. A. (1987). Competitive interactions within and between species in a guild of avian scavengers. The Auk, 104(2), 290-295. https://doi. org/10.1093/auk/104.2.290

Zak, M. R., Cabido, M., Cáceres, D. y Díaz, S. (2008).
What drives accelerated land cover change in central Argentina? Synergistic consequences of climatic, socioeconomic, and technological factors. Environmental Management, 42(2), 181-189. https://doi. org/10.1007/s00267-008-9101-y

\section{Material suplementario}

https://rdu.unc.edu.ar/handle/11086/16788

\section{NOTA}

1.- En Argentina, se refiere a la casa donde viven el encargado o los trabajadores de un campo. 